\title{
A random walker on a ratchet potential: Effect of a non Gaussian noise
}

\author{
Sergio E. Mangioni $[\dagger]$ \\ Departamento de Física, FCEyN, \\ Universidad Nacional de Mar del Plata \\ Deán Funes 3350, \\ 7600 Mar del Plata, Argentina \\ Horacio S. Wio \\ Instituto de Física de Cantabria, \\ Universidad de Cantabria and CSIC, \\ Avda. Los Castros $s / n$, \\ E-39005 Santander, Spain
}

\begin{abstract}
We analyze the effect of a colored non Gaussian noise on a model of a random walker moving along a ratchet potential. Such a model was motivated by the transport properties of motor proteins, like kinesin and myosin. Previous studies have been realized assuming white noises. However, for real situations, in general we could expect that those noises be correlated and non Gaussian. Among other aspects, in addition to a maximum in the current as the noise intensity is varied, we have also found another optimal value of the current when departing from Gaussian behavior. We show the relevant effects that arise when departing from Gaussian behavior, particularly related to current's enhancement, and discuss its relevance for both biological and technological situations.
\end{abstract}

PACS numbers: 05.40.Jc, 82.20.Uv,05.40.-a 


\section{INTRODUCTION}

Noise induced transport by Brownian motors or "ratchets" has attracted the attention of an increasing number of researchers due to its biological interest as well as its potential technological applications. Since the pioneering works, besides the built-in ratchet-like bias and correlated fluctuations, several different aspects have been studied, such as tilting [1, 2] and pulsating [3] potentials, velocity inversions [1, 4], etc. There are relevant reviews [5, 6] indicating the biological and/or technological motivations for the study of ratchets as well as showing the state of the art.

Among other aspects, ratchets has been used to explain the unidirectional transport of molecular motors within a biological realm [5, 6]. Among the different motor proteins, kinesin has attracted considerable attention motivated by experimental results in which the dynamical details of its motion can be measured [7, 8, 9]. Kinesin is a protein with two heads that performs a walk along the microtubule inside cells. Motivated by these experimental results, several researchers have introduced diverse models in order to understand the particular form of walking of kinesin [10]. Usually those models consider a walker moving along an asymmetric ratchet potential mediated by noise. This walker has two feet that are represented by two particles nonlinearly coupled through a bistable potential. Among those models, there is a recent one introduced in [11, 12] including all the above indicated ingredients, where the walker moves along a track formed by an asymmetric potential, being subjected to two independent white noise sources acting on each of the two particles and to a common external harmonic force. It was observed that the current $J$ as function of noise intensity $D_{w}$ presents a maximum, conforming another example of the constructive role of noise.

Recent studies on the effect of a non Gaussian noise on several noise induced phenomena, have shown the existence of strong effects on the system's response. In those studies, opposing to the most usual cases that only consider Gaussian white noises, such an effect was analyzed in stochastic resonance, noise induced transitions, "standard" ratchets, etc [13, 14, 15, 16, 17]. This form of noise was motivated by the nonextensive statistical mechanics [18, 19]. In this work we want to analyze the effect of this form of colored and non Gaussian noise over the kinesin ratchet model introduced in Refs. [11, 12]. For this purpose, we used a mean field approximation and exploited a recently developed technique [17]. 
Through the variation of a parameter $q$, this form of noise offers the possibility of analyzing the departure from Gaussian behavior (corresponding to $q=1$ ). Since subtle change of environment conditions can produce drastic changes in biological process, such effects could be very relevant. It is worth here indicating that a related mean field approximation was introduced in [20].

The organization of the paper is as follows. In the next Section we show the mean-field approximation and how reliable it is, based on the good agreement with known results for the Gaussian case. The third Section presents the statistical properties of the non Gaussian noise, and the form of the current that results after applying the effective Markovian approximation in the present problem. In the following Section we present the results when we depart from the Gaussian behavior, that is varying the parameter $q$. Finally, in the last Section we present some conclusions.

\section{THE MEAN-FIELD APPROXIMATION AND ITS ACCURACY: GAUSSIAN CASE}

The stochastic dimensionless differential equations for the two particles in the overdamped regime, whose coordinates are indicated by $x$ and $y$, are [11]

$$
\begin{aligned}
& \dot{x}=-\partial_{x} V(x)-\partial_{x} V_{b}(x-y)+\sqrt{2 D_{w}} \xi_{1}(t)+A \sin \Omega t, \\
& \dot{y}=-\partial_{y} V(y)-\partial_{y} V_{b}(x-y)+\sqrt{2 D_{w}} \xi_{2}(t)+A \sin \Omega t,
\end{aligned}
$$

where $V(x)$ is the dimensionless ratchet potential

$$
V(x)=C+U_{R}\left[\sin \left(2 \pi\left(x-x_{0}\right)\right)-0.25 \sin \left(4 \pi\left(x-x_{0}\right)\right)\right]
$$

The constant $C=U_{R}\left[\sin \left(2 \pi x_{0}\right)-0.25 \sin \left(4 \pi x_{0}\right)\right]$ was chosen in such a way that $V(0)=0$. The constant $x_{0}$ is introduced in order to center the minima of the periodic potential on integer values. The dimensionless amplitude of the ratchet potential is indicated by $U_{R}$.

The dimensionless bistable potential $V_{b}(x-y)$, that represents the nonlinear coupling between the two particles, is given by

$$
V_{b}(x-y)=U_{b}\left[1+\frac{(x-y)^{4}}{l^{4}}-2 \frac{(x-y)^{2}}{l^{2}}\right],
$$

where $U_{b}$ is the dimensionless amplitude of this bistable potential and $2 l$ is the distance between the two minima. 
In the original model, it is assumed that $\xi_{1}(t)$ and $\xi_{2}(t)$ are Gaussian white noises with zero mean and correlation $\left\langle\xi_{i}(t) \xi_{i}\left(t^{\prime}\right)\right\rangle=2 D_{w} \delta_{i, j} \delta\left(t-t^{\prime}\right)$, with $D_{w}$ the intensity of the statistically independent noises. In our model, as indicated at the introduction, we assume they are non Gaussian colored noises, with characteristics that we will briefly indicated later. Clearly, the model also considers an external harmonic force.

In [12] this model was analytically solved. However, in order to consider the non Gaussian colored noise case, we need to introduce a different approach. For that purpose, we consider a mean field approximation (MFA) [21]. For the present case, such a MFA consist in the following approximation for $V_{b}(x-y)$

$$
V_{b}(x-M)=U_{b}\left[1+\frac{(x-M)^{4}}{l^{4}}-2 \frac{(x-M)^{2}}{l^{2}}\right],
$$

with $M=\langle y\rangle$, and clearly we have $\langle x\rangle=\langle y\rangle$. After applying the MFA, the equations for both variables $x$ and $y$ results to be of the same form. Hence, we can reduce the problem to a single equation describing the model system

$$
\dot{x}=-\partial_{x} V_{e f f}(x, M, t)+\sqrt{2 D_{w}} \xi(t),
$$

where $V_{\text {eff }}(x, M, t)=V(x)+V_{b}(x-M)-x A \sin \Omega t$.

In order to test the MFA, we start analyzing the Gaussian white case, and show that it gives similar qualitative results than the numerical simulations and the analytical solution for the original model [11, 12]. Hence, in Eq. (44), we start assuming that $\xi(t)$ is a Gaussian white noise with the same behavior than the $\xi_{i}(t)$ 's.

Considering an adiabatical approximation in order to decouple the external harmonic force from the rest, the corresponding Fokker-Planck equation can be solved assuming periodic boundary conditions. Even though $V_{\text {eff }}(x, M, t)$ is non periodic [due to $V_{b}(x-M)$ being non periodic], we can still assume such a periodic behavior since both feet are never too far away from each other, the strong slope of the attractive potential preventing it to occur. In Fig. 1 it is shown $V_{b}(x)$ vs. $x$ with and without the indicated approximation. In Fig. 2 we show $V_{\text {eff }}(x)$ vs. $x$, again with and without the approximation. It is apparent that the curves looks quite alike.

Considering the adiabatical approximation, the corresponding stationary solution of the Fokker-Planck equation is [22]

$$
P^{s t}(x, M)=\frac{H(x)}{N \sqrt{2 D_{w}}} e^{-V_{e f f}(x, M) / D_{w}},
$$




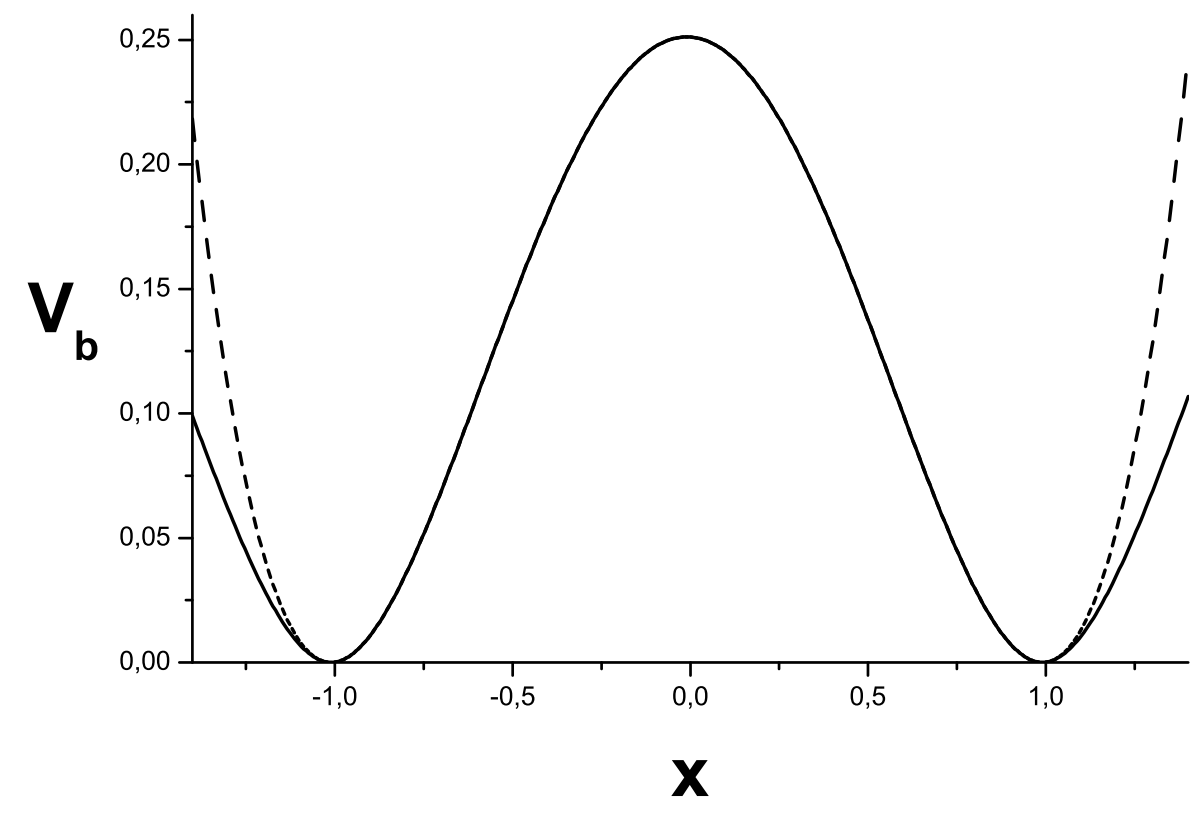

FIG. 1: The bistable potential $V_{b}$ vs. $x$. The continuous line corresponds to the exact form of the potential, while the dashed line is for the approximate one. The parameters are $U_{b}=0.2512$ and $l=1$.

with

$$
H(x)=\frac{1}{\sqrt{2 D_{w}}} \int_{x}^{x+L} d u e^{V_{e f f}(u, M) / D_{w}},
$$

$N$ a normalization constant, and $L=2 \pi$ the period. Hence, numerically solving

$$
\int_{-L / 2}^{L / 2} d x x P^{s t}(x, M)=M=<x>
$$

we can obtain the current $J(t)[22]$ as

$$
J(t)=\frac{1}{2 N}\left[1-e^{\left(V_{e f f}(L)-V_{e f f}(0)\right) / D_{w}}\right]
$$

and the net current is obtained as

$$
J=\frac{1}{T} \int_{0}^{T} J(t) d t
$$

with $T=\frac{2 \pi}{\Omega}$.

In order to check these results in Fig. 3 we depict $J$ vs. $D_{w}$. The curve presents a maximum for an "optimal" value of the noise intensity, which is qualitatively similar to the one obtained in [11, 12]. With the support offered by this agreement, we are now in position to use this same approach for the non Gaussian colored noise case. 


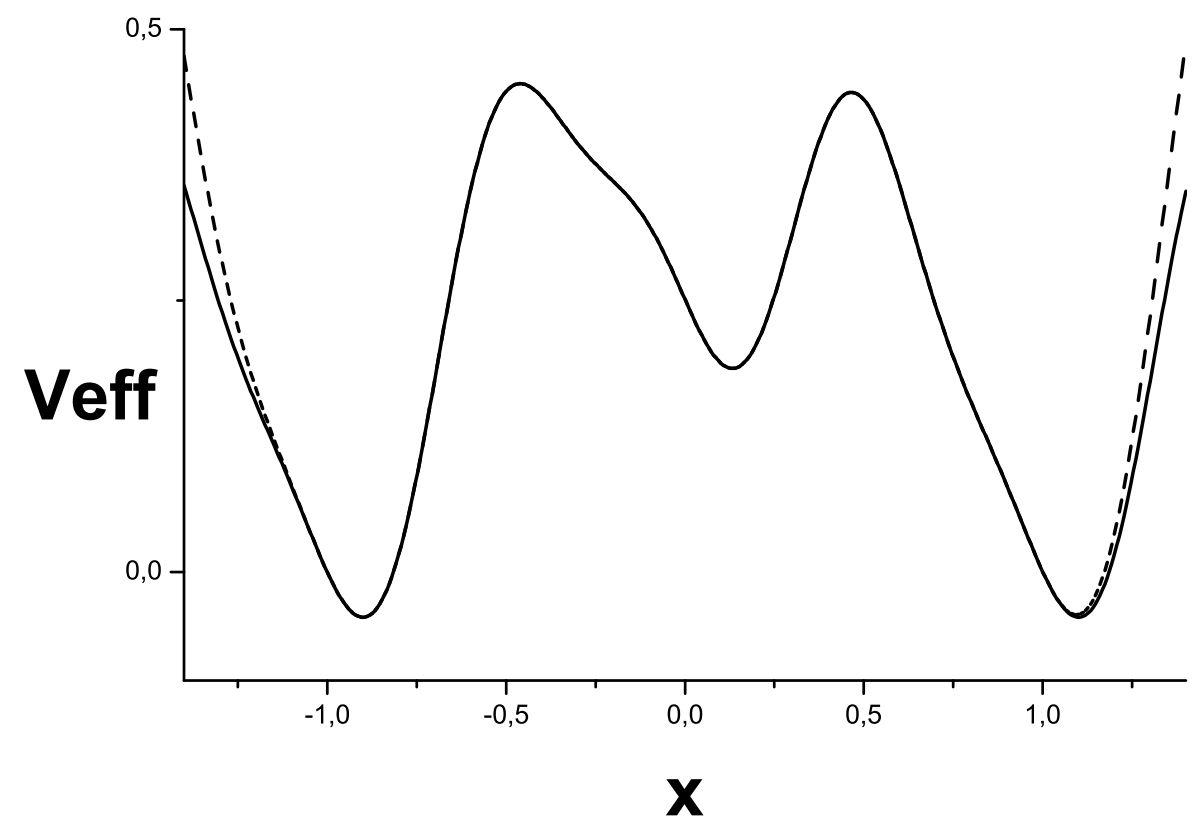

FIG. 2: Effective potential $V_{\text {eff }}$ vs. $x$. The continuous line corresponds to the exact form of the potential, while the dashed line is for the approximate one. The parameters are $A=1, l=1$, $U_{r}=0.16, U_{b}=0.2512$ and $t=0$.

\section{NON GAUSSIAN NOISE CASE}

\section{A. Statistical properties of the non Gaussian noise}

We consider now the non Gaussian colored noise case as in [13]. Hence, in Eq. (41) $\xi(t)$ is a noise with a dynamics described by the following Langevin equation

$$
\dot{\xi(t)}=-\frac{1}{\tau} \frac{d}{d \xi)} V_{q}(\xi)+\frac{1}{\tau} \eta(t)
$$

with $\eta(t)$ a white Gaussian noise, and

$$
V_{q}(\xi)=\frac{D_{w}}{\tau(q-1)} \ln \left[1+\frac{\tau}{D_{w}(q-1) \frac{\xi^{2}}{2}}\right]
$$

For $q=1$, the process $\xi$ coincides with the Ornstein-Uhlenbeck (OU) one (with a correlation time equal to $\tau$ ), while for $q \neq 1$ it departs from the Gaussian behavior. For $q<1$ the stationary probability distribution (spd) has a bounded support, with a cut-off given by 


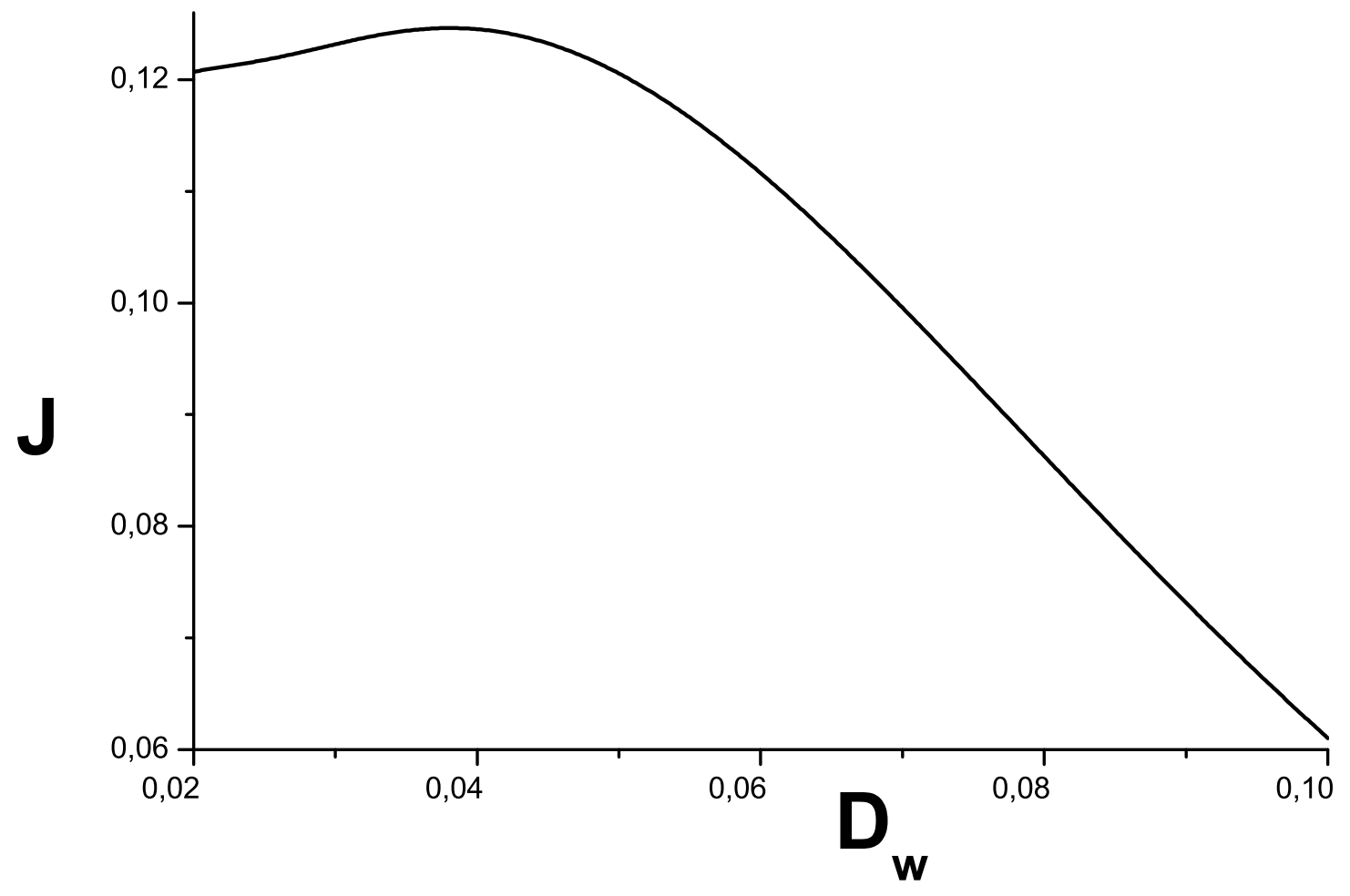

FIG. 3: Net current $J$ for a gaussian white noise, as function of $D_{w}$. The values of parameters are $A=1, l=1, U_{r}=0.16$ and $U_{b}=0.2512$.

$\|\xi\|=\omega \equiv\left[(1-q) \tau /\left(2 D_{w}\right)\right]^{-\frac{1}{2}}$, with a form given by

$$
P_{q}(\xi)=\frac{1}{Z_{q}}\left[1-\left(\frac{\xi}{\omega}\right)^{2}\right]^{\frac{1}{1-q}},
$$

for $\|\xi\|<\omega$ and zero for $\|\xi\|>\omega\left(Z_{q}\right.$ is a normalization constant). Within the range $1<q<3$, the spd is given by

$$
P_{q}(\xi)=\frac{1}{Z_{q}}\left[1+\frac{\tau(q-1) \xi^{2}}{2 D_{w}}\right]^{\frac{1}{1-q}},
$$

for $-\infty<\xi<\infty$, and decays as a power law (that is, slower than a Gaussian distribution) for $\xi \rightarrow \infty$. Finally, for $q>3$, this distribution can not be normalized. Note that keeping $D_{w}$ constant, when increasing $q$ the dispersion of the distribution also increases. In [13] the second moment of the distribution was obtained. This moment is related to the intensity of 
the non Gaussian noise, and is given by

$$
D_{n g}=<\xi^{2}>=\frac{2 D_{w}}{\tau(5-3 q)},
$$

that diverges for $q \geq 5 / 3$. For $\tau_{n g}$, the correlation time of the process $\xi(t)$, that was defined in detail in [13], it was not possible to find an analytical expression. However, it is known [13] that for $q \rightarrow 5 / 3$ it diverges as $(5-3 q)^{-1}$. In [13] $\tau_{n g}$ vs. $q$ was numerically calculated for the range $0.5<q<5 / 3$. The following analytical approximation, accurate within the indicated range, was found

$$
\tau_{n g}=\frac{2 \tau}{(5-3 q)}\left[1+4(q-1)^{2}\right]
$$

\section{B. Current in the non Gaussian case}

The Fokker-Planck equation for the present case, that is the system described by the Langevin equation indicated by Eq. (44) with $\xi(t)$ the non Gaussian noise, has a known structure. After applying the effective Markovian approximation as exploited in [13, 15, 17], such an equation has the form

$$
\partial_{t} P(x, t)=\partial_{x}[A(x) P(x, t)]+\frac{1}{2} \partial_{x}^{2}[B(x) P(x, t)]
$$

where

$$
A(x)=\frac{V_{e f f}^{\prime}}{A_{1}(x)+A_{2}(x)}
$$

with

$$
\begin{aligned}
A_{1}(x) & =\frac{1-\left(\tau / 2 D_{w}\right)(q-1) V_{e f f}^{\prime 2}}{1+\left(\tau / 2 D_{w}\right)(q-1) V_{e f f}^{\prime 2}} \\
A_{2}(x) & =\tau V_{e f f}^{\prime \prime}\left[1+\left(\tau / 2 D_{w}\right)(q-1) V_{e f f}^{\prime 2}\right]
\end{aligned}
$$

and

$$
B(x)=D_{w}\left[\frac{\left[1+\left(\tau / 2 D_{w}\right)(q-1) V_{e f f}^{\prime 2}\right]^{2}}{B_{1}(x)+B_{2}(x)}\right]^{2}
$$

with

$$
\begin{aligned}
& B_{1}(x)=\tau V_{e f f}^{\prime \prime}\left[1+\left(\tau / 2 D_{w}\right)(q-1) V_{e f f}^{\prime 2}\right]^{2} \\
& B_{2}(x)=\left[1-\left(\tau / 2 D_{w}\right)(q-1) V_{e f f}^{\prime 2}\right] .
\end{aligned}
$$

The prima indicates a derivative respect to $x$. 
The corresponding stationary solution of the indicated Fokker-Planck equation is [22]

$$
P^{s t}(x)=\frac{e^{-\phi(x)}}{N S(x)} \int_{x}^{x+L} d u \frac{e^{\phi(u)}}{S(u)},
$$

with $N$ a normalization constant, and

$$
\phi(x)=-\int_{0}^{x} d u \frac{R(u)}{S^{2}(u)},
$$

where $S(x)=\sqrt{B}$ and $R(x)=-A-\frac{1}{2} S S^{\prime}$. Then, using Eqs. (7), as (8) and (9) we can obtain the current $J$.

\section{RESULTS}

In what follows we present several results for the case of submitting the system to a non Gaussian noise, mainly restricting ourselves to the range $0.5<q<1.5$, which is the range where our evaluation is, in principle, valid. In all cases, except we indicated something different, we adopted $\tau_{n g}=0.01 \pi, A=1, l=1, \Omega=0.5, U_{r}=0.16$ and $U_{b}=0.2512$.

In Fig. 4 we show the dependence of $J$ on $q$, for different values of $D_{n g}$. Looking at the left part of the picture, we identify the curves, from top to bottom, with the values $D_{n g}=0.02$, 0.038, 0.06, 0.07, 0.085, 0.1 and 0.13. The entanglement between the dependence on $q$ and $D_{n g}$ is apparent. For low values of $D_{n g}\left(D_{n g} \sim 0.02\right)$ the behavior of $J$ corresponds to an initial plateau for $q<1$ and a fast (step like) decrease for $q>1$. When $D_{n g}$ increases $\left(D_{n g} \gtrsim 0.06\right), J(q)$ adopts a bell's shape, with a maximum that shifts to larger values of $q$ for increasing $D_{n g}$. It is worth noting that for large values of $D_{n g}$, the relevant values for the current will only occur when $q$ takes values within the indicated bell-like region.

As in previous studies on the effect of non Gaussian noise on other systems [13, 14, 15, 16, 17] we conclude that the observed change of behavior is associated with the process $\xi(t)$, that is with the departure from Gaussian behavior $(q=1)$ and the associated changes in $P_{q}(\xi)$. As indicated before, for $q<1$ there is a cut-off that prevents that $\xi(t)$ reaches large values, while for $q>1 \xi(t)$ can reach very large values. For small values of $D_{n g}$ it is expected that the current $J(q)$ will not be very sensitive to such a cut-off, as the probability for $\xi(t)$ to reach those cut-off values is very small. In opposition, for large values of $D_{n g}$ we expect that $J(q)$ becomes highly sensitive to the cut-off. It seems to be the case as $J$ varies very slowly with $q$ (for $q<1$ ) when $D_{n g}$ is small, while for large values of $D_{n g}$ just the opposite occurs. 


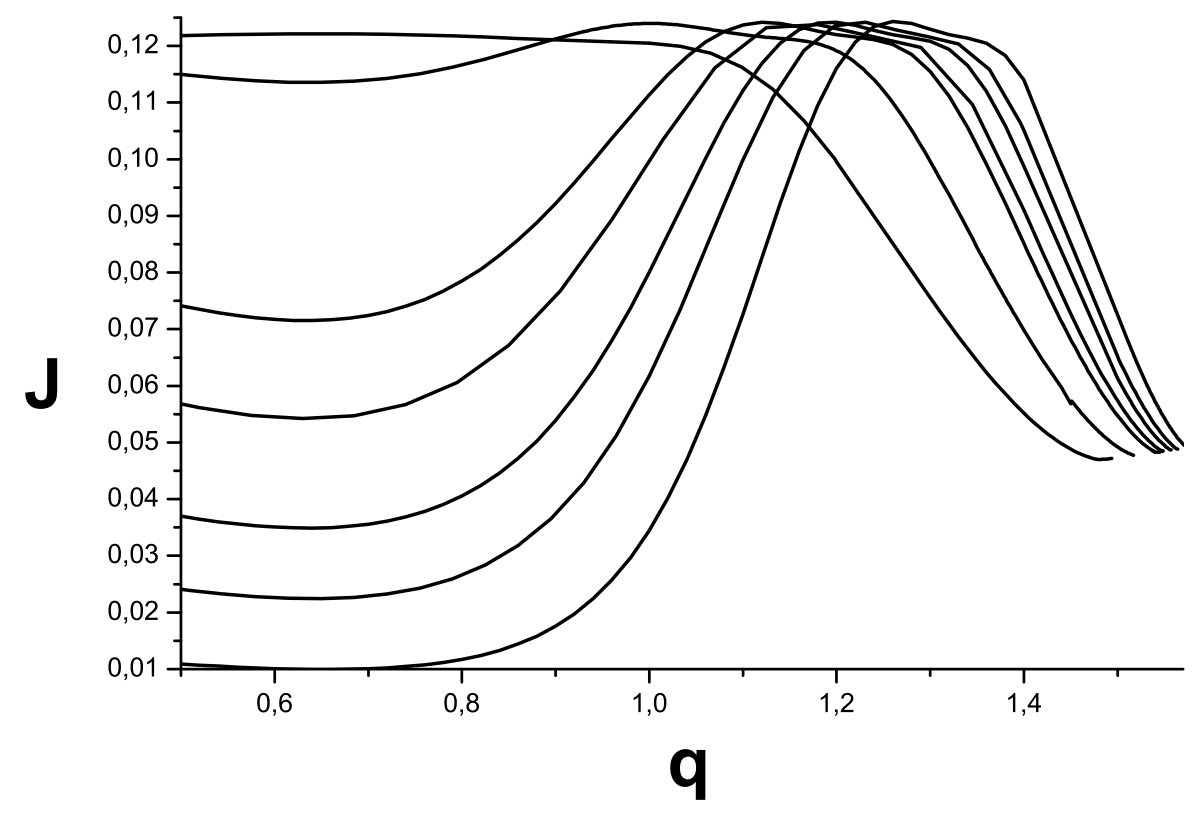

FIG. 4: Net current $J$ vs. $q$, for the range $.5<q<1.5$. Looking at the left part of picture, we identify from top to bottom $D_{n g}=0.02,0.038,0.06,0.07,0.085,0.1$ and 0.13 . Other parameters are: $\tau_{n g}=0.01 \pi, A=1, l=1, U_{r}=0.16$ and $U_{b}=0.2512$.

However, for $q>1$, it is the tail of the spd that mainly contributes to build the current [17]. As indicated above, for $q<1$ (that is, for the case without tail), and large values of $D_{n g}, J$ drastically decreases when $q$ decreases. Let us select one of the curves for $J$ with a bell shape, and look into the spd for values of $q$ below, at, and above of the maximum of that curve. Such analysis is shown in Fig. 5. We have chosen $D_{n g}=0.085, q=0.94$ (below), $q=1.2$ (at the maximum) and $q=1.4$ (above). We observe that for the case of the maximum, the spd is symmetric with two peaks at both ends of the space period. However, when departing from such "optimal" condition, the spd looses its symmetry. In one hand, when $q$ is at the left of the maximum $(q=0.94)$ a new peak emerges at the central zone of the period. On the other hand, when $q$ is to right of the maximum, there are two peaks that arise between the previously indicated ones. In both cases, the prize of these additional peaks is that the spd decreases at both ends, indicating a reduction of $J$. The same behavior was also observed in all cases where $J(q)$ adopts a bell's shape. We also compare the spd behavior for the case when $J(q)$ has a step-like shape, and note that 


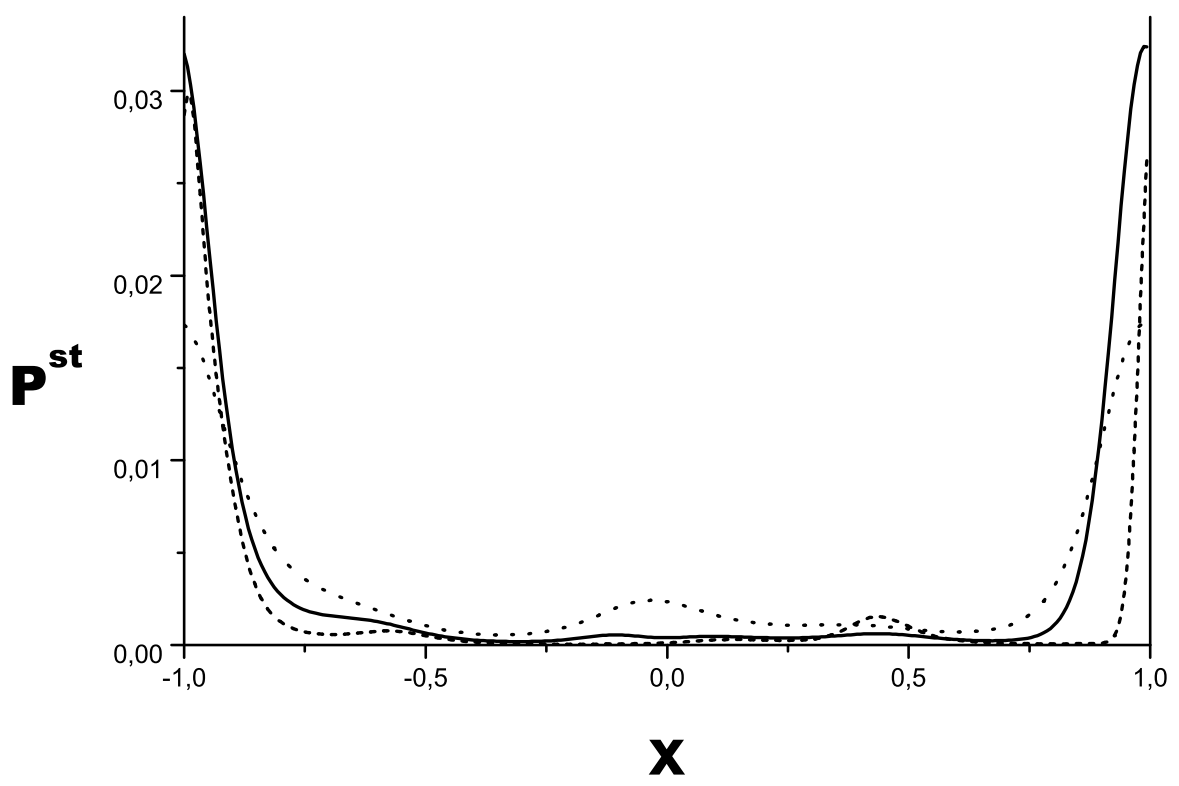

FIG. 5: Stationary probability distribution $P^{s t}$ for $q$ below, at, and after of maximum $J$ 's value, with $D_{n g}=0.085$ and: dot line for $q=0.94$, solid line for $q=1.2$ and dash line for $q=1.4$. Other parameters are: $\tau_{n g}=0.01 \pi, A=1, l=1, U_{r}=0.16$ and $U_{b}=0.2512$.

for values of $q$ within the plateau, the spd is like the one corresponding to the case of the maximum of $J(q)$ (Fig. 3, for $q=1.2$ ), while for values of $q$ beyond the step, the spd is analogous to the one corresponding to a value of $q$ located at the right of the maximum $(q=1.4)$. It seems that the current reaches a maximum when one feet follows the other, and their separation is kept constant, and equal to a period. We have also analyzed the Gaussian case $(q=1)$ for different values of $D_{n g}$, with results that essentially reproduces those of Fig. 5. In short, for small $D_{n g}$ the curves are analogous to those obtained for large $q$ (having four peaks), while for large $D_{n g}$ the shapes resembles the ones for small $q$ (having three peaks).

Figure 6 depicts $J(t)$-the current before its time averaging- as function of time, for a fixed $D_{n g}$ and different values of $q$. It is apparent that the current adopts both, positive and negative values along the period of the external forcing. We observe that for $q<q_{\max }$ (where $J\left(q_{\max }\right)$ reaches its largest values) there is a balance between positive and negative current's values yielding, in average, a small net current. When $q$ increases, the negative values of the current reduces, the indicated balance is also reduced, and the net current 


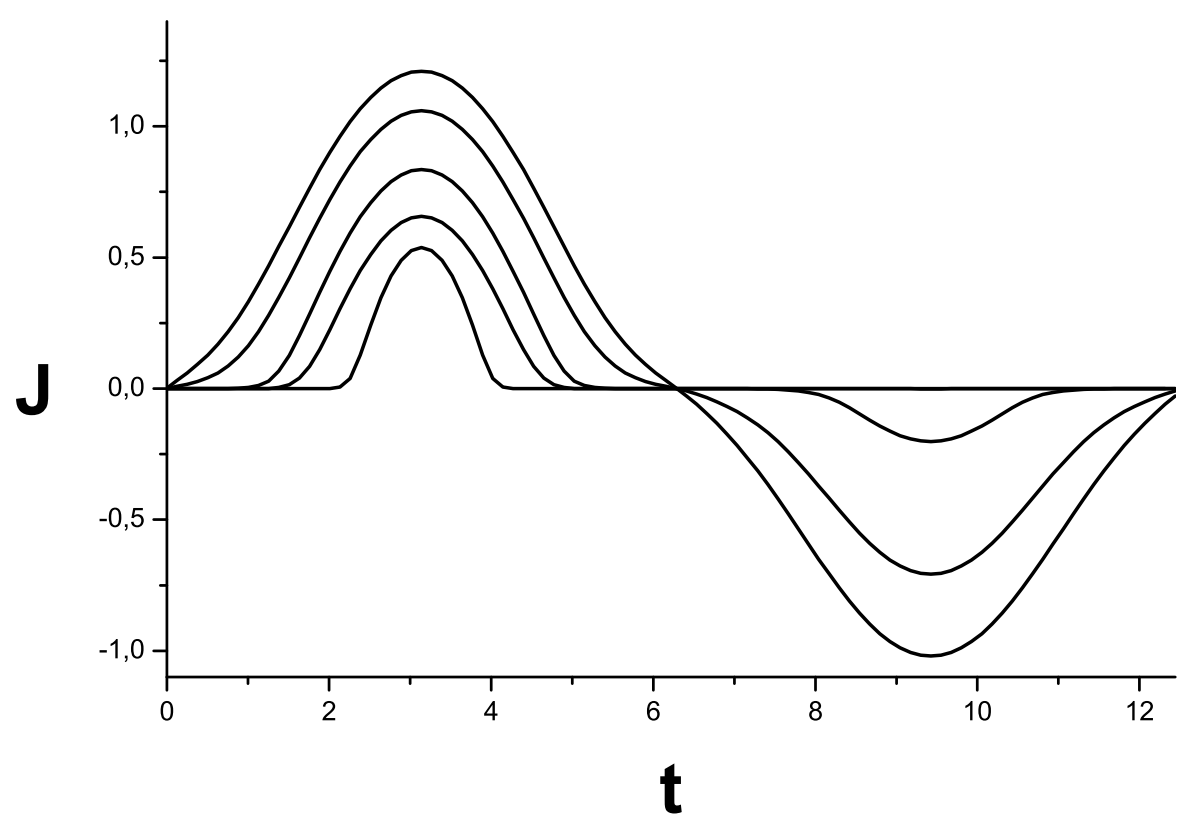

FIG. 6: $J(t)$, the current before the time averaging, vs. $t$, for $D_{n g}=0.085$ and different values of $q$ 's. Adopting as reference the left part of the figure, from top to bottom we have $q=0.3,1 ., 1.2$, 1.4 and 1.55. Other parameters are: $\tau_{n g}=0.01 \pi, A=1, l=1, U_{r}=0.16$ and $U_{b}=0.2512$.

increases. For even larger values of $q\left(q>q_{\max }\right)$, the positive value of the current is reduced, while the negative one tend to zero, and the net current decreases.

We finally analyze the system's behavior with $\tau_{n g}$. Firstly, we observed that inside the studied range, $J$ varies almost linearly with $\tau_{n g}$. Since the corresponding slope change its sign with $q$ and $D_{n g}$, we choose to look into the behavior of $d J / d \tau_{n g}$. Hence, in Fig. 7 we show $d J / d \tau_{n g}$ vs. $q$ for different values of $D_{n g}$, while in Fig. 8 we show $d J / d \tau_{n g}$ vs. $D_{n g}$ for different values of $q$. The sensitivity of $d J / d \tau_{n g}$ against variations of $q$ and $D_{n g}$ is apparent, and the changes in the slope's sign are clearly seen.

\section{CONCLUSIONS}

In line with some recent work [13, 14, 15, 16, 17], we have here analyzed the effect of a colored and non Gaussian noise over the kinesin ratchet model introduced in [11, 12]. As was discussed in [13], there are strong experimental evidences that noise sources within 


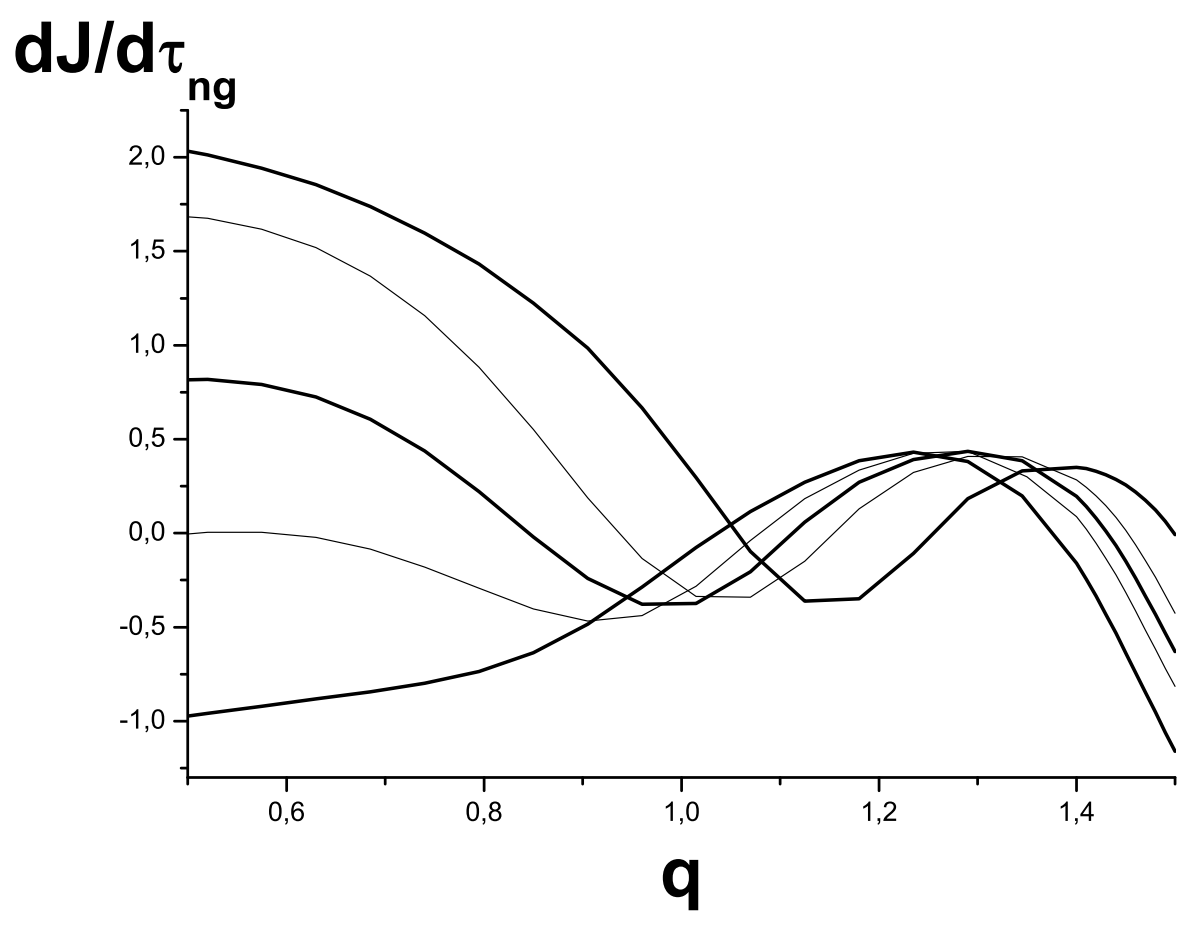

FIG. 7: $d J / d \tau_{n g}$ vs. $q$ for different $D_{n g}$. Considering the left part of the figure, we identify from top to bottom, $D_{n g}=0.1,0.05,0.038,0.03$ and 0.02 . Other parameters are: $\tau_{n g}=0.01 \pi, A=1$, $l=1, U_{r}=0.16$ and $U_{b}=0.2512$.

biological systems can not be in general Gaussian [23]. This fact gives strong support to the study of the effect of non Gaussian noises within a biological motivated context.

In order to make an almost analytical treatment, we have here exploited a mean-field like approach, that shows a nice agreement when it is tested against known results [11, 12] for the Gaussian case $(q=1)$. Hence, we can trust the results we have obtained when varying the parameter $q$. For the adopted form of noise, such variation offers the possibility of analyzing the departure $(q \neq 1)$ from the Gaussian behavior $(q=1)$.

When analyzing the dependence of the current $J$ on $D_{n g}$ and $q$, we have confirmed the existence, for a fixed value of $q$, of a maximum or "optimal" current as function of $D_{n g}$. What is new, is that for fixed $D_{n g}$, there is also an optimal value of $q$ yielding a maximum value of the current. More, in general such a value corresponds to a non Gaussian situation $(q \neq 1)$. Such a results could be understood from the behavior of the spd for different values of $q$, as well as from the current's time dependence (that is, before the time averaging). 


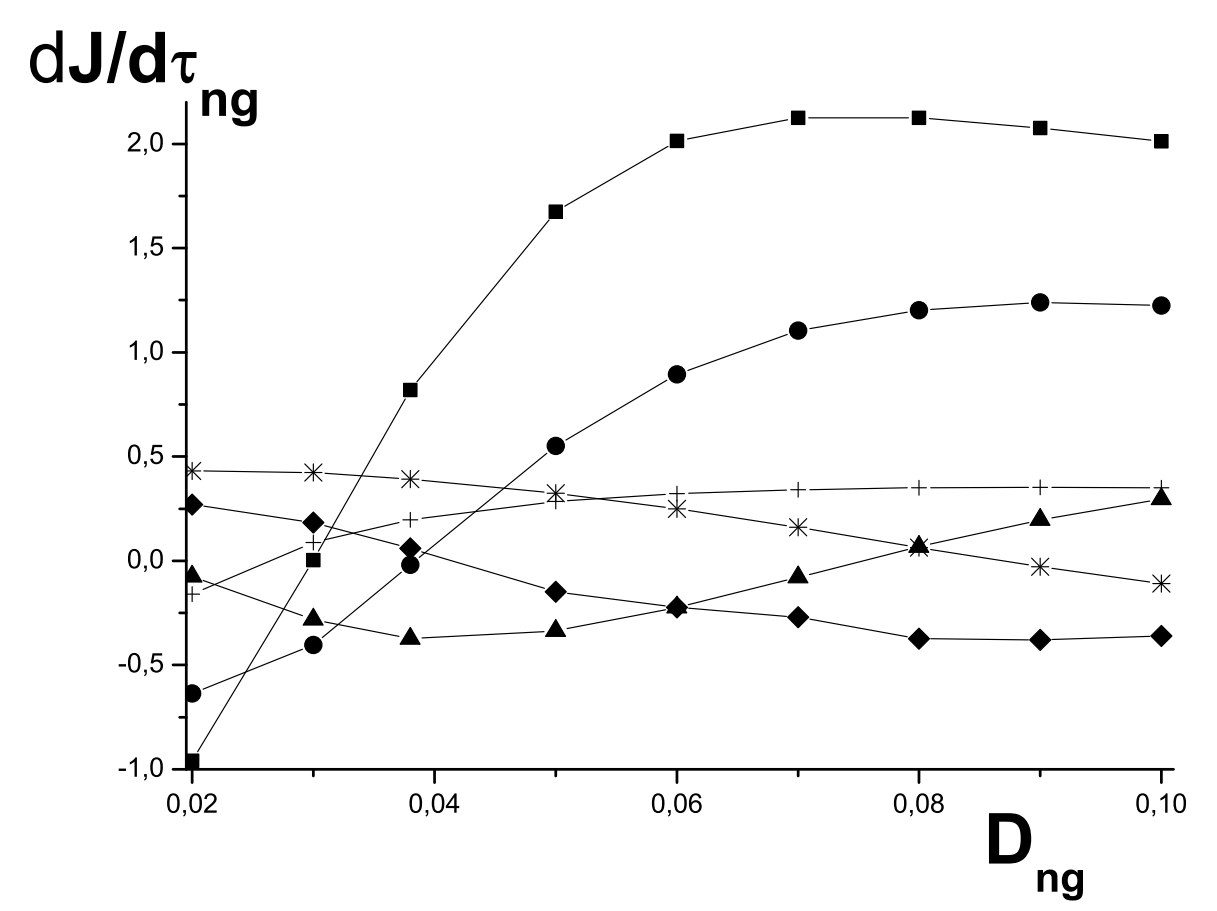

FIG. 8: $d J / d \tau_{n g}$ vs. $D_{n g}$ for different values of $q$ : squares $q=0.52$, circles $q=0.85$, triangles $q=1.015$, diamonds $q=1.125$, stars $q=1.235$ and crosses $q=1.4$. Other parameters are: $\tau_{n g}=0.01 \pi, A=1$, $l=1, U_{r}=0.16$ and $U_{b}=0.2512$.

Regarding the dependence of $J$ on $\tau_{n g}$, we have seen that it is linear. However, analyzing its slope $\left(d J / d \tau_{n g}\right)$ it becomes apparent the possibilities of even changing the sign of such a slope varying both, $q$ for fixed $D_{n g}$, or $D_{n g}$ for fixed $q$.

The indicated results show us the richness of behavior that we can found when departing from the Gaussian situation. It is apparent that those results could be of relevance not only for biological studies, but for technological applications as well. This point was discussed in general in [16], and particularly for the case of ratchets in [17]. From a technological point of view, such a noise source offers a variety of forms of controlling and/or optimizing the transport process.

Clearly, the exploitation of an approach like the one used in [12], based in a coordinate separation, could offer a more transparent and better description of this system's physics. In addition a numerical validation of the whole approach is required. Also, another aspect that requires further analysis is the case when $2 l$ is non-commensurate with the unit cell of 
the ratchet potential. All these aspects will be the subject of forthcoming work [24].

\section{Acknowledgments}

SEM acknowledges financial support from CONICET, Argentina, PIP 5072, and from UNMdP, EXA 338/06. HSW acknowledges financial support from MEC, Spain, through Grant No. CGL2004-02652/CLI, and thanks the European Commission for the award of a Marie Curie Chair during part of the development of this work.

[†] Member of CONICET, Argentine.

[1] C. R. Doering, W. Horsthemke, and J. Riordan, Phys. Rev. Lett. 72, 2984 (1994).

[2] M.Millonas and M.I Dykman, Phys. Lett. A 185, 6 (1994); R. Bartussek, P. Hänggi and J. G. Kissner, Europhys. Lett. 28, 459 (1994).

[3] P. Reimann and P. Hänggi, in Lect Notes in Physics, vol. 484 (Springer, Berlin 1997).

[4] F. Marchesoni, Phys. Lett. A. 237, 126 (1998); J. L. Mateos, Phys. Rev. Lett. 84, 258 (2000).

[5] R. D. Astumian, Science 276, 917 (1997).

[6] P. Reimann, Phys. Rep. 361, 57 (2002); P. Reimann and P. Hänggi, Appl. Phys. A 75, 169 (2002); P. Hänggi, F. Marchesoni and F. Nori, Ann. Phys. (Leipzig) 14, 51 (2005).

[7] R.D. Vale and R.A. Milligan, Science 288, 88 (2000).

[8] D. Keller and C. Bustamante, Biophys. J. 78, 541 (2000).

[9] J. Howard, Mechanics of Motor Proteins and the Cytoskeleton (Sinauer Ass. Sunderland, MA, 2001).

[10] I. Derenyi and T. Vicsek, Proc. Natl. Acad. Sci. USA, 93, 6775 (1996); G.N. Stratopoulos, T.E. Dialynas and G.P. Tsironis, Phys. Lett. A 252, 151 (1999); S. Klumpp, E. Mielke and C. Wald, Phys. Rev. E 63,031914 (2001); M. Bier, Phys. Rev. Lett. 91, 148104 (2003); D. Dan, A.M. Jayannavar and G.I. Menon, Physica A 318, 40 (2003); H.-Y. Wang and J.-D. Bao, Physica A 337, 13 (2004).

[11] J.L. Mateos, Physica A, 351, 79 (2005)

[12] J. Menche and L. Schimansky-Geier, Phys. Lett. A, 359, 90 (2006).

[13] M.A. Fuentes, R. Toral and H.S. Wio, Physica A 295, 114-122 (2001); M.A. Fuentes, H.S. 
Wio and R. Toral, Physica A 303, 91 (2002); M.A. Fuentes, C. Tessone, H.S. Wio and R. Toral, Fluctuations and Noise Letters 3, L365 (2003); F.J. Castro, M.N. Kuperman, M.A. Fuentes and H.S. Wio, Phys. Rev. E 64, 051105 (2001).

[14] H.S. Wio, J.A. Revelli and A.D. Sánchez, Physica D 168-169, 165 (2002).

[15] H.S. Wio and R. Toral, in Anomalous Distributions, Nonlinear Dynamics and Nonextensivity, H. Swineey and C. Tsallis (Eds.), Physica D 193, 161 (2004).

[16] H.S. Wio, On the Role of Non-Gaussian Noises, chapter in Ref. ([19]); H.S. Wio, Noise induced phenomena and nonextensivity, Special issue, Europhys. News, 197 (Nov-Dec 2005).

[17] S. Bouzat and H.S. Wio, Eur. Phys. J. B 41, 97 (2004); S. Bouzat and H.S. Wio, Physica A 351, 69 (2005).

[18] C. Tsallis, Stat. Phys. 52, 479 (1988); E.M.F. Curado and C. Tsallis, J. Phys. A 24, L69 (1991); ibidem, 24, 3187 (1991); ibidem, 25, 1019 (1992).

[19] M.Gell-Mann and C. Tsallis (Eds.), Nonextensive Entropy-Interdisciplinary Applications (Oxford U.P., Oxford, 2003).

[20] S. Savel'ev, F. Marchesoni and F. Nori, Phys. Rev. Lett. 91, 010601 (2003) and Phys. Rev. Lett. 92, 160602 (2004); S. Savel'ev, F. Marchesoni, P. Hänggi and F. Nori, Phys. Rev. E 70, 066109 (2004).

[21] S. Mangioni, R. Deza, H. S. Wio and R. Toral, Phys. Rev. Lett. 79, 2389 (1997); S. E. Mangioni, R. R. Deza, R. Toral and H. S. Wio, Phys. Rev. E 61, 223 (2000); S. E. Mangioni, R. R. Deza and H.S. Wio, Phys. Rev. E 63, 041115 (2001); H. S. Wio, S. E. Mangioni and R. R. Deza, Physica D 168-169C, 186 (2002); S. E. Mangioni, R. R. Deza and H. S. Wio, Phys. Rev. E 66, 051106 (2002).

[22] C.W. Gardiner, Handbook of Stochastic Methods, 2nd Ed. (Springer-Verlag, Berlin, 1985); N.G. van Kampen, Stochastic Processes in Physics and Chemistry (North Holland, Amsterdam, 2004).

[23] S. M. Bezrukov and I. Vodyanoy, Nature 378, 362 (1995); D. Nozaki, D.J. Mar, P. Griegg and J.D. Collins, Phys. Rev. Lett. 72, 2125 (1999); A. Manwani and C. Koch, Neural Comp. 11, 1797 (1999); A. Manwani, Information-Theoretic Analysis of Neuronal Communication, PhD Thesis, CALTECH, (2000).

[24] S. E. Mangioni and H. S. Wio, in preparation. 